\title{
Food insecurity and maternal depression in rural, low-income families: a longitudinal investigation
}

\author{
Catherine Huddleston-Casas ${ }^{1}$, Richard Charnigo ${ }^{2}$ and Leigh Ann Simmons ${ }^{3, *}$ \\ 'Department of Child, Youth and Family Studies, University of Nebraska-Lincoln, Omaha Campus, 102 Arts and \\ Sciences Hall, Omaha, NE 68182-0214, USA: ${ }^{2}$ College of Public Health 203-B, Department of Biostatistics, \\ University of Kentucky, Lexington, KY 40536-0003, USA: ${ }^{3}$ Departments of Family Studies and Health Services \\ Management, University of Kentucky, 315 Funkhouser Building, Lexington, KY 40506-0054, USA
}

Submitted 3 March 2008: Accepted 11 August 2008: First published online 15 September 2008

\begin{abstract}
Objective: The purpose of the present study was to examine the relationship between household food insecurity and maternal depression in a rural sample to determine whether food insecurity predicted mothers' depression over time or vice versa.

Design: The study employed a prospective design using three waves of data from 'Rural Families Speak', a multi-state study of low-income rural families in the USA. Food insecurity was measured using the Core Food Security Module and depression was measured using the Center for Epidemiologic Studies-Depression Scale. A structural equation model was fit to the data using the AMOS software package. Setting: Sixteen states in the USA (California, Indiana, Kentucky, Louisiana, Massachusetts, Maryland, Michigan, Minnesota, Nebraska, New Hampshire, New York, Ohio, Oregon, South Dakota, West Virginia, Wyoming) between 2000 and 2002.

Subjects: Subjects included 413 women with at least one child under the age of 13 years living in the home.

Results: Findings based on the 184 subjects with complete data indicated that the causal relationship between household food insecurity and depression is bidirectional $(P=0.034$ for causation from depression to food insecurity, $P=0.003$ for causation from food insecurity to depression, $\chi^{2} / \mathrm{df}=1 \cdot 835$, root-mean-square error of approximation $=0 \cdot 068$, comparative fit index $=0 \cdot 989$ ). Findings based on all 413 subjects after imputation of missing values also indicated bidirectionality.

Conclusions: The recursive relationship between food insecurity and depression has implications for US nutrition, mental health and poverty policies. The study highlights the need to integrate programmes addressing food insecurity and poor mental health for the population of rural, low-income women.
\end{abstract}

Keywords Food insecurity Depression Rural women United States
Poverty is a significant social problem in the USA that is associated with a number of public health concerns, including poor mental and physical health status and disparities in health care $^{(1)}$. The most recent statistics show the national poverty rate increased to $9.8 \%$ between 2000 and $2006^{(2)}$. In rural areas this rate is even higher, with recent data showing that the rural poverty rate exceeds $14 \%{ }^{(3)}$. These data reflect an ongoing trend of poverty and related problems faced by rural Americans over the last 50 years ${ }^{(4)}$.

One of the problems associated with rural poverty is food insecurity, which is defined as being uncertain of having or unable to acquire enough food to meet the needs of all family members due to insufficient money or other resources ${ }^{(5)}$. The rate of food insecurity nationwide increased from $9 \cdot 8 \%$ to $11 \cdot 0 \%$ between 2001 and $2006^{(6)}$, and the rural food insecurity rate reached $12 \%$ in $2006^{(6)}$. The prevalence of food insecurity varies among household types. Households headed by single mothers and households with incomes below the federal poverty line have higher rates of food insecurity than those in the general population $^{(6)}$. Minority households also have a higher rate of food insecurity. Recent data revealed Black and Latino households to have twice the rate of food insecurity as White households. Similarly, households with children were almost twice as likely to be food-insecure as households without children $(15 \cdot 6 \% v .8 \cdot 5 \%)^{(6)}$. In rural areas food insecurity is particularly concerning, because rural residents have more limited access to affordable food than their urban counterparts. Compared with urban consumers, rural residents face higher prices for food $^{(7)}$ and are less likely to receive food stamps despite eligibility ${ }^{(8)}$. 
A growing body of research has demonstrated a link between food insecurity and depression, although most of these studies were cross-sectional in nature. A study of California women showed that poor mental health was linked to food insecurity, but the authors noted that the direction of effect could not be determined ${ }^{(9)}$. In a qualitative study of poor women in Philadelphia, Chilton and Booth ${ }^{(10)}$ found that food-insecure women experienced 'hunger of the mind', which they defined as a sense of hopelessness and depression. In a study of women with young children, Casey and colleagues ${ }^{(11)}$ found that nearly $53 \%$ of women who reported food insecurity scored positive on a depression screen. Similarly, a crosssectional study of nearly 3000 mothers of 3-year-olds showed that the percentage of respondents with major depressive disorder increased with greater levels of food insecurity. Among food-secure mothers, $15 \cdot 7 \%$ had major depressive disorder, compared with $20 \cdot 2 \%$ of marginally food-secure mothers and $28.5 \%$ of food-insecure mothers. This research is underscored by findings from the Women's Employment Study, which revealed that urban welfare recipients in Michigan who experienced food insufficiency also were more likely to experience depression $^{(12-14)}$. As other researchers have noted, the authors of the Michigan studies acknowledged that the direction of effect could not be ascertained.

The relationship between food insecurity and depression is concerning in light of the high rates of depression among rural women. Although assessments of the prevalence of depressive symptomatology and major depressive episodes (MDE) among rural populations are limited, Hauenstein and colleagues ${ }^{(15)}$ argue that population mental health deteriorates as the level of rurality increases. The prevalence of clinically depressive symptomatology among rural women has been shown to range from $24 \%$ to $69 \%^{(16-22)}$, and low-income women appear to be particularly vulnerable ${ }^{(16-18,21,22)}$.

Given the high prevalence rates of both food insecurity and depression among rural women, and given previous studies suggesting that food insecurity and depression are associated, the purpose of the present study was to examine the longitudinal relationship between food insecurity and depression in a sample of low-income, rural women. Since previous studies have failed to document a direction of effect between food insecurity and depression, our goal was to examine whether: (i) food insecurity predicts depression; (ii) depression predicts food insecurity; or (iii) the relationship between food insecurity and depression is bidirectional. Additionally, to our knowledge this is the first longitudinal study of the relationship between food insecurity and depression among rural, low-income women in the USA. Findings have the potential to affect public policy and practice aimed at reducing the rates of both food insecurity and depression in this vulnerable population.

\section{Methods}

\section{Sample}

We utilized data from NC-223, 'Rural Families Speak' (RFS), a multi-state (California, Indiana, Kentucky, Louisiana, Massachusetts, Maryland, Michigan, Minnesota, Nebraska, New Hampshire, New York, Ohio, Oregon, South Dakota, West Virginia, Wyoming) study of rural, low-income women and their families. The study was approved by a research ethics board at all participating institutions, and all respondents provided informed consent. Between 2000 and 2002, three waves of data were collected in twenty-three rural counties on an annual basis at each participating research site. Investigators defined rural using Butler and Beale's ${ }^{(23)}$ coding system for metropolitan and non-metropolitan areas. Of the participating counties, seventeen were coded as 6 (population of 2500 to 19 999, adjacent to a metropolitan area), 7 (population of 2500 to 19 999, not adjacent to a metropolitan area) or 8 (completely rural or less than 2500 urban population, adjacent to a metropolitan area). The remaining six counties were in states without counties having Beale codes of 6-8. Four counties were in rural areas with no urban centres of populations exceeding 10000 , and two counties had no population centre greater than 10000 .

A convenience sample of 413 respondents was recruited in wave 1 from programmes and places serving lowincome families, including Welfare-to-Work programmes, Head Start programmes, the Special Supplemental Nutrition Program for Women, Infants, and Children (WIC), public health clinics, and low-income housing. This approach to sample selection allowed the research team to access this hard-to-reach population through contacts known to and trusted by the participants. Trust is an extremely important issue for families in rural communities where anonymity and privacy are difficult to acquire and maintain. Thus, convenience sampling was preferable over randomized sampling. To be eligible for participation, women had to be at least 18 years of age, have at least one child aged 13 years or younger, and be eligible for food stamps in their state. All data were collected via an interviewer-administered questionnaire typically lasting $1 \cdot 5-2 \cdot 0 \mathrm{~h}$. Of the original 413 respondents interviewed at wave 1, 325 were re-interviewed at wave 2 and 270 were re-interviewed at wave 3 for an overall attrition rate of $34 \cdot 6 \%$.

\section{Measures}

Food insecurity was measured at all three waves by the Core Food Security Module (CFSM), an eighteen-item scale with a 12-month time reference administered as part of the Current Population Survey conducted by the US Census Bureau for the Bureau of Labor Statistics. The scale has good validity and reliability, with a reliability coefficient of $\alpha=0.81$ for households with children ${ }^{(24)}$. 
The CFSM is similarly reliable for populations of racial and ethnic minorities ${ }^{(25)}$. Reliability for this rural sample matched reported reliability $(\alpha=0 \cdot 81)$.

Depression was measured at all three waves using the Center for Epidemiologic Studies-Depression Scale (CES-D), which was designed to measure depressive symptomatology in the general population ${ }^{(26)}$. The measure includes twenty items, each rated on a 4-point scale ranging from 'rarely' to 'most or all of the time'. Respondents are asked to report on how they have been feeling over the last week. Statements include: (i) 'I felt depressed'; (ii) 'I felt everything I did was an effort'; and (iii) 'I felt lonely'. There are four subscales, which include depressed affect, negative affect, interpersonal relationships and somatic symptoms. The four questions for negative affect are framed positively and then reverse scored. Reliability for the CES-D is $\alpha=0.85$ in the general population and $\alpha=0.90$ in psychiatric populations. Reliability in this sample matched the reliability reported for the general population $(\alpha=0 \cdot 85)$.

Five sociodemographic variables also were measured at wave 1, including age, ethnicity, household income, martial status and education. Age and household income were measured continuously. Martial status was measured in five categories (single, married, living with partner, divorced, separated). Ethnicity was measured in four categories (White, Black, Latina, other). Education was measured in six categories (8th grade or less, some high school, high school degree or general education equivalent, technical training, some college/associate's degree, college degree or higher).

\section{Data analysis}

Since the food insecurity scores at each wave had a positively skewed distribution, we applied a logarithmic transformation to these scores before carrying out the following analyses. Bivariate associations among the depression scores and food insecurity scores were assessed using Pearson correlations. Each Pearson correlation was based on all subjects for whom measurements on both variables were available; the number of such subjects varied according to which two variables were considered. We then formulated a structural equation model encompassing all three waves of depression scores and food insecurity scores. A schematic for the model is presented in Fig. 1. The coefficients $\alpha_{1}$ and $\alpha_{2}$ link a depression score to a subsequent depression score, $\alpha_{3}$ and $\alpha_{4}$ link a food insecurity score to a subsequent food insecurity score, $\alpha_{5}$ and $\alpha_{6}$ link a depression score to a subsequent food insecurity score, and $\alpha_{7}$ and $\alpha_{8}$ link a food insecurity score to a subsequent depression score.

We fit two versions of the structural equation model. The primary version was based on the 184 subjects for whom depression scores were recorded at all three waves (i.e. were not missing at any wave), food insecurity scores were recorded at all three waves, and income was recorded at the first wave. We required the availability of income for a test of whether $\alpha_{1}$ to $\alpha_{8}$ were the same across the two median-determined income strata, as previous work ${ }^{(27)}$

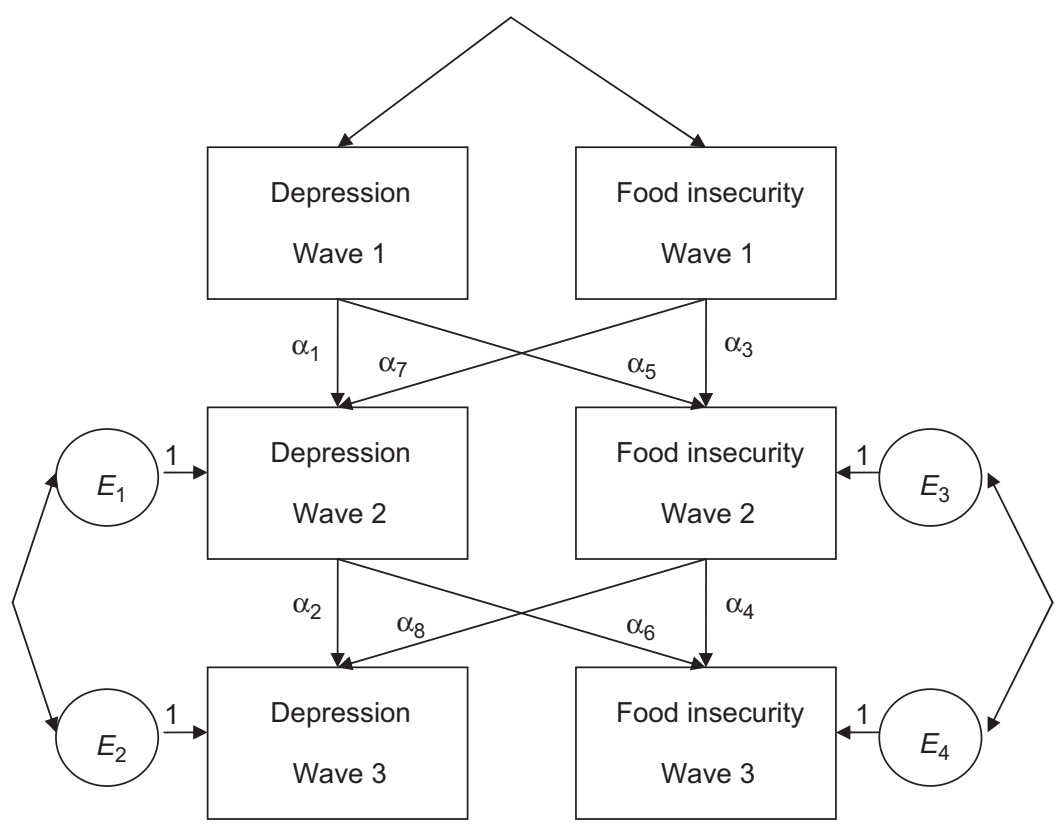

Fig. 1 Schematic for the structural equation model. Depression at wave 2 was modelled as a function of depression at wave 1, food insecurity at wave 1 , and an error variable $E_{1}$. Depression at wave 3 was modelled as a function of depression at wave 2 , food insecurity at wave 2 , and an error variable $E_{2}$. Food insecurity at wave 2 was modelled as a function of depression at wave 1 , food insecurity at wave 1 , and an error variable $E_{3}$. Food insecurity at wave 3 was modelled as a function of depression at wave 2 , food insecurity at wave 2 , and an error variable $E_{4}$. Depression and food insecurity at wave 1 were assumed to be correlated, as were the two error variables affecting depression and the two error variables affecting food insecurity. The coefficients $\alpha_{1}$ to $\alpha_{8}$ were estimated using the structural equation modelling capabilities of AMOS version 7 
had documented a significant association between income and depression scores. Besides testing whether the coefficients were the same across income strata, we tested two other null hypotheses. One of these was that $\alpha_{5}=\alpha_{6}=0$, meaning that depression would not impact food insecurity; the other was that $\alpha_{7}=\alpha_{8}=0$, meaning that food insecurity would not impact depression.

The secondary version of the structural equation model was based on all 413 subjects, with missing values estimated by regression imputation prior to fitting the model. Since standard errors of coefficient estimates are inversely related to the square root of the sample size, the secondary version of the structural equation model should offer more precise, although slightly biased, coefficient estimates. All analyses were carried out using the Statistical Package for the Social Sciences version 15 and AMOS version 7 statistical software packages (SPSS Inc., Chicago, IL, USA).

Three complementary fit statistics were utilized to evaluate the structural equation models. First is the ' $\chi^{2} / \mathrm{df}$ lackof-fit statistic. The $\chi^{2}$ value pertains to a null hypothesis that the population covariance matrix of the observed variables equals the covariance matrix implied by the model. Because this null hypothesis is generally presumed to be false, most researchers do not formally accept or reject it. Rather, they divide the $\chi^{2}$ value by its degrees of freedom. A large quotient $(>5)$ indicates serious lack of fit and a small quotient $(<3)$ suggests little lack of fit ${ }^{(28)}$. Second is the root-meansquare error of approximation (RMSEA) lack-of-fit statistic, which is a non-negative quantity expressing the discrepancy between the population covariance matrix and that implied by the model. A large RMSEA $(>0 \cdot 10)$ reflects serious lack of fit, while a small RMSEA $(<0 \cdot 05)$ implies little lack of $\mathrm{fit}^{(28)}$. Third is the comparative fit index (CFI) goodness-of-fit statistic, which is a quantity between 0 and 1 describing how much the model improves upon a straw-man 'independence model'. A large CFI $(>0 \cdot 95)$ indicates good fit ${ }^{(29)}$.

\section{Results}

Demographic data have been summarized elsewhere ${ }^{(27)}$. Briefly, the majority of respondents were married or living with a partner, non-Hispanic White, and had a high school education, general education equivalent, or less. The mean age of participants was $30 \cdot 1$ years and median household income was $\$ 14826$. There was a significant association between income and depression scores, but depression scores were not significantly associated with marital status, race, education or age.

Pearson correlations for the depression scores and food insecurity scores are presented in Table 1 . All of the correlations were positive and significantly different from 0 (all $P<0 \cdot 01)$. Particularly strong correlations were found between wave 1 depression scores and wave 2 depression scores (0.534), between wave 2 depression scores and wave 3 depression scores $(0 \cdot 620)$, and between any two sets of food insecurity scores $(0.524,0 \cdot 540,0 \cdot 547)$.

The results for the primary version of the structural equation model, supposing that $\alpha_{1}$ to $\alpha_{8}$ are the same across income strata, are summarized in the 'Complete case analysis' columns of Table 2 . The estimates of $\alpha_{1}, \alpha_{2}$, $\alpha_{3}, \alpha_{4}$ and $\alpha_{7}$ were positive and significantly different from 0 (all $P<0 \cdot 001$ ). The estimates of $\alpha_{5}, \alpha_{6}$ and $\alpha_{8}$ were not significantly different from 0 (all $P>0 \cdot 10$ ). However, coefficient estimates based on a sample size as small as 184 are fairly imprecise, so non-significant estimates do not imply that the coefficients equal 0 . More importantly, a test of the null hypothesis $\alpha_{5}=\alpha_{6}=0$ yielded $\chi^{2}=6.754$ on 2 df $(P=0 \cdot 034)$. Thus, while we cannot make the specific claim that $\alpha_{5} \neq 0$ and cannot make the specific claim that $\alpha_{6} \neq 0$, we can infer that at least one of these two coefficients is non-zero. Hence, the model cannot be simplified by omitting the links from depression to food insecurity, which is to say that there is a causal relationship from depression to food insecurity. In addition, a test of the null hypothesis $\alpha_{7}=\alpha_{8}=0$ yielded $\chi^{2}=11.786$ on $2 \mathrm{df}(P=0 \cdot 003)$. Therefore, the model cannot be simplified by omitting the links from food insecurity to depression, which is to say that there is a causal relationship from food insecurity to depression. In sum, the model asserts a bidirectional relationship between food insecurity and depression, and fit statistics support this: $\chi^{2} / \mathrm{df}=1 \cdot 835$, RMSEA $=0 \cdot 068$, CFI $=0 \cdot 989$. Following the guidelines in the data analysis section, we

Table 1 Pearson correlations for depression and food insecurity: rural, low-income women with at least one child $<13$ years of age living in the home, USA, 2000-2002

\begin{tabular}{|c|c|c|c|c|c|c|}
\hline & $\begin{array}{l}\text { Depression } \\
\text { Wave } 1\end{array}$ & $\begin{array}{l}\text { Depression } \\
\text { Wave } 2\end{array}$ & $\begin{array}{c}\text { Depression } \\
\text { Wave } 3\end{array}$ & $\begin{array}{c}\text { Food insecurity } \\
\text { Wave } 1\end{array}$ & $\begin{array}{c}\text { Food insecurity } \\
\text { Wave } 2\end{array}$ & $\begin{array}{c}\text { Food insecurity } \\
\text { Wave } 3\end{array}$ \\
\hline Depression Wave 1 & 1 & 0.534 & 0.383 & 0.305 & $0 \cdot 270$ & $0 \cdot 227$ \\
\hline Depression Wave 2 & 0.534 & 1 & 0.620 & 0.335 & $0 \cdot 292$ & $0 \cdot 344$ \\
\hline Depression Wave 3 & 0.383 & 0.620 & 1 & $0 \cdot 246$ & $0 \cdot 229$ & $0 \cdot 321$ \\
\hline Food insecurity Wave 1 & $0 \cdot 305$ & $0 \cdot 335$ & $0 \cdot 246$ & 1 & 0.524 & 0.540 \\
\hline Food insecurity Wave 2 & $0 \cdot 270$ & $0 \cdot 292$ & $0 \cdot 229$ & 0.524 & 1 & 0.547 \\
\hline Food insecurity Wave 3 & $0 \cdot 227$ & $0 \cdot 344$ & $0 \cdot 321$ & 0.540 & 0.547 & 1 \\
\hline
\end{tabular}

Pearson correlations were calculated, using SPSS version 15, to quantify the bivariate associations for all pairs of variables that could be chosen from among depression at wave 1, depression at wave 2, depression at wave 3, food insecurity at wave 1, food insecurity at wave 2 , and food insecurity at wave 3 . Each Pearson correlation was based on all subjects for whom measurements on both variables were available. All Pearson correlations were significantly different from 0 at the $0 \cdot 01$ level. 
Table 2 Structural equation modelling results for the relationship between depression and food insecurity: rural, low-income women with at least one child $<13$ years of age living in the home, USA, 2000-2002

\begin{tabular}{|c|c|c|c|c|c|c|c|c|}
\hline \multirow[b]{2}{*}{ Coefficient } & \multicolumn{4}{|c|}{ Complete case analysis } & \multicolumn{4}{|c|}{ Analysis with missing values imputed } \\
\hline & Estimate & SE & $\begin{array}{l}\text { Standardized } \\
\text { estimate }\end{array}$ & $P$ & Estimate & SE & $\begin{array}{l}\text { Standardized } \\
\text { estimate }\end{array}$ & $P$ \\
\hline$\alpha_{1}$ & $0 \cdot 426$ & 0.061 & $0 \cdot 442$ & $<0.001$ & 0.468 & 0.036 & 0.528 & $<0.001$ \\
\hline$\alpha_{2}$ & $1 \cdot 015$ & $0 \cdot 146$ & $0 \cdot 858$ & $<0.001$ & $0 \cdot 870$ & 0.064 & 0.853 & $<0.001$ \\
\hline$\alpha_{3}$ & 0.486 & 0.060 & 0.505 & $<0.001$ & 0.500 & 0.036 & 0.565 & $<0.001$ \\
\hline$\alpha_{4}$ & $1 \cdot 034$ & 0.144 & 1.053 & $<0.001$ & 0.911 & 0.073 & 0.962 & $<0.001$ \\
\hline$\alpha_{5}$ & 0.006 & 0.004 & 0.077 & 0.132 & 0.006 & 0.003 & 0.082 & 0.023 \\
\hline$\alpha_{6}$ & 0.008 & 0.005 & $0 \cdot 103$ & $0 \cdot 102$ & $0 \cdot 011$ & 0.003 & $0 \cdot 139$ & $<0.001$ \\
\hline$\alpha_{7}$ & $2 \cdot 436$ & 0.708 & 0.212 & $<0.001$ & $2 \cdot 103$ & 0.437 & 0.193 & $<0.001$ \\
\hline$\alpha_{8}$ & -0.826 & 0.885 & -0.058 & 0.350 & -0.379 & 0.477 & -0.030 & 0.427 \\
\hline
\end{tabular}

For the structural equation model depicted in Fig. 1, the coefficients $\alpha_{1}$ to $\alpha_{8}$ were estimated using AMOS version 7. The results under 'Complete case analysis' are based on the 184 subjects for whom there were no missing values on depression or food insecurity and for whom income at wave 1 was not missing. The results under 'Analysis with missing values imputed' are based on all 413 subjects, with any missing values imputed using AMOS version 7 prior to fitting the structural equation model. Each $P$ value pertains to a null hypothesis that the corresponding coefficient is 0 .

determined that the structural equation model adequately represents the empirical patterns among depression scores and food insecurity scores, and the assertion of a bidirectional relationship is credible.

Finally, to justify the supposition that $\alpha_{1}$ to $\alpha_{8}$ are the same across income strata, we refit the model allowing $\alpha_{1}$ to $\alpha_{8}$ (along with variance/covariance parameters) to be estimated separately for subjects with wave 1 income below the median (\$14 826) and for subjects with wave 1 income above the median. We then tested the null hypothesis that $\alpha_{1}$ to $\alpha_{8}$ (along with variance/covariance parameters) are the same across income strata. We obtained $\chi^{2}=18 \cdot 074$ on 17 df $(P=0 \cdot 384)$, which justifies the supposition.

The results for the secondary version of the structural equation model are summarized in the 'Analysis with missing values imputed' columns of Table 2. The estimates of $\alpha_{1}, \alpha_{2}, \alpha_{3}, \alpha_{4}, \alpha_{5}, \alpha_{6}$ and $\alpha_{7}$ were positive and significantly different from 0 ( $P$ for $\alpha_{5}=0.023$, all other $P<0 \cdot 001)$. The estimate of $\alpha_{8}$ was not significantly different from $0(P=0 \cdot 427)$. A test of the null hypothesis $\alpha_{5}=\alpha_{6}=0$ yielded $\chi^{2}=23.558$ on $2 \mathrm{df}(P<0 \cdot 001)$, while a test of the null hypothesis $\alpha_{7}=\alpha_{8}=0$ yielded $\chi^{2}=22.636$ on $2 \mathrm{df}(P<0 \cdot 001)$. Hence, the secondary version of the model also supports the conclusion of a bidirectional relationship between food insecurity and depression, and model fit statistics confirm this: $\chi^{2} / \mathrm{df}=$ $2 \cdot 454$, RMSEA $=0 \cdot 059, \mathrm{CFI}=0 \cdot 994$. These statistics suggest that the secondary version of the model adequately captures the empirical patterns among depression scores and food insecurity scores, adding further credibility to the conclusion of a bidirectional relationship.

\section{Discussion}

The present study found evidence of a simultaneous causal relationship between food insecurity and depression in a sample of rural, low-income women. To our knowledge, this study is the first of its kind to examine longitudinally the relationship between food insecurity and depression in the USA. This finding is important, not only because it underscores previous research showing food insecurity and depression are closely related, but also because it demonstrates the relationship is recursive. Thus, interventions aimed at reducing the prevalence of food insecurity in low-income rural households and depression in women living in these households can be approached on multiple levels.

Interventions aimed at increasing household food security in rural areas must target barriers systemically, including addressing high prices and increasing access to food stamps. Rural supermarket prices are on average $4 \%$ higher than in suburban areas ${ }^{(7)}$. Further, there are fewer supermarkets and more small grocery stores, the latter of which have prices averaging 10\% higher than chain supermarkets nationwide. Low-income rural residents are particularly vulnerable to paying higher prices for food. Thus, tax incentives should be provided to major supermarket chains to establish a presence in rural areas with low supermarket penetration.

Beyond paying higher prices for food, one-third of rural residents eligible to receive food stamps do not participate in the programme ${ }^{(8)}$, which further limits access. Food stamp programmes should include campaigns to educate rural residents about eligibility requirements and increase access to this important resource. Further, research suggests that the effectiveness of food stamp redemption could be increased. A US Department of Agriculture analysis of food stamp redemptions in low-income, rural areas revealed that supermarkets accounted for only $52 \cdot 8 \%$ of redemptions, compared with $76 \cdot 7 \%$ of redemptions in supermarkets nationwide ${ }^{(7)}$. These data suggest that rural families receiving food stamps are more likely than their urban counterparts to spend these benefits in smaller grocery stores where food is more expensive, thus decreasing the buying power of the stamps. Support for strategies to maximize the value of food stamps is needed. Travel vouchers to offset the expense of travelling to a supermarket in a neighbouring community in conjunction with 
budgeting and meal planning would assist families in making the most of food stamp participation.

Given the recursive relationship between food insecurity and depression, other possible interventions might occur at the level of the food stamp nutrition education programmes (FSNEP). In light of data demonstrating that deficiencies in vitamin $\mathrm{B}_{6}^{(30)}$ and folic acid ${ }^{(31,32)}$ contribute to depressive illness, FSNEP could educate participants on how to economically increase their intakes of these nutrients. Further, FSNEP could develop and test brief depression screening and referral programmes. Such programmes might improve identification of depression in a population whose members are less likely to be seen for mental health problems than their urban and suburban counterparts ${ }^{(33)}$. Screening for maternal depression in other non-traditional settings has been shown to be beneficial $^{(34)}$.

As with food insecurity, research shows that rural women living in low-income households are vulnerable to depression, specifically undiagnosed depression. Hauenstein and Boyd ${ }^{(16)}$ found that $41 \cdot 4 \%$ of 181 rural women interviewed by telephone reported clinically depressive symptomatology on the CES-D. Within their sample, young, unemployed and poorly educated women were most likely to report depressive symptoms. Sears et al. $^{(17)}$ assessed depression in 136 rural, lowincome, predominantly female primary care patients using the Primary Care Evaluation of Mental Disorders (PRIME-MD) and found that $46 \%$ screened positive for depression; fifty-three of those sixty-three patients (40\% of the total sample) were further evaluated and classified as depressed. A qualitative investigation of the daily hassles faced by thirty-three rural, poor single mothers found that nearly $69 \%$ of the sample reported clinically depressive symptomatology (CES-D scores of 16 or greater $^{(18)}$. Hauenstein and Peddada ${ }^{(21)}$ sampled 982 impoverished rural women attending a community health centre for a routine visit and found approximately half screened positive for depressive symptoms. Follow-up assessments revealed that $14.3 \%$ of the positive screeners had current MDE.

Despite these high prevalence rates, rural residents often do not receive treatment. Research shows that rural residents with a history of depression are less likely to seek professional help ${ }^{(35,36)}$. Further, stigma ${ }^{(37,38)}$ and lack of anonymity ${ }^{(39)}$ prevent many rural residents from seeking psychological care. These social barriers are compounded by structural barriers, including the shortage of qualified mental health professionals in rural areas and the lack of service outreach ${ }^{(33)}$. Additionally, Hauenstein $^{(37)}$ found that even when care is available, rural women may not access it, because traditional mental health care is not congruent with how women discuss and understand their depression. Thus, mental health interventions in rural areas might include education to de-stigmatize depression and its treatments (both pharmacological and talk therapy) as well as programmes to increase access to mental health care, such as mobile mental health services and tele-health programmes. Further, given the simultaneous relationship between food insecurity and depression, mental health care providers in rural areas might include as part of their psychosocial evaluation an assessment of food security and dietary status. Such screenings may identify poor nutritional intake as an important contributor to poor mental health status and result in improved access to important food supports, such as food stamps.

\section{Limitations}

Findings from the present study must be considered within the context of its limitations. Although these data highlight the complexity of the relationship between food insecurity and depression over time in an under-researched population, this sample is not nationally representative, thus limiting generalizability to the broader rural population in the USA. Further, because the sample consists of rural residents only, the recursive relationship between food insecurity and depression found here may not extend to urban/suburban populations. Additionally, although results were similar for the primary model (missing data excluded) and the secondary model (missing data imputed), there is always the possibility of bias with incomplete data. Similarly, bias is also possible with self-reported data despite the use of standardized instruments to measure food insecurity and depression.

\section{Conclusions}

The present study highlights the importance of food security to good mental health and the importance of good mental health to food security. This is especially relevant for professionals serving populations that are vulnerable to both food insecurity and depression, such as rural low-income women. Resources directed at the health and well-being of such populations may be made more effective by simultaneously addressing nutrition and mental health. Programmes directed at the food security and nutrition needs of low-income families could address the potential influence of depression on food insecurity by assessing the need for mental health services. Individuals requiring mental health care could be referred to appropriate providers or programmes. Reciprocally, programmes and services designed to enhance the mental health of rural low-income families could include food security and nutritional health evaluations as part of their psychological intake assessment. Mental health providers treating individuals confronting depression as well as food insecurity and nutrition issues could refer their clients to the appropriate programmes and services. Public health nutritionists would benefit from education about the association between food insecurity and depression 
within rural populations. Additionally, these professionals could work with mental health professionals to simultaneously and comprehensively address the consequences of poor nutrition and poor mental health in rural settings. Future research focused on complementary nutrition and mental health interventions is warranted.

\section{Acknowledgements}

This research was supported in part by USDA/CSREES/ NRICGP Grant 2001-35401-10215, 2002-35401-11591 and 2004-35401-14938, and by NIH/NIDA Grant 5 K12 DA014040-07 (to L.A.S.). The opinions expressed herein are solely those of the authors. There are no additional conflicts of interest. C.H.-C. contributed to the writing of the manuscript. R.C. conducted the analyses and contributed to the writing. L.A.S. conceptualized the study, contributed to the writing, and assisted with primary data collection for the 'Rural Families Speak' project.

\section{References}

1. Probst JC, Ladtika SB, Moore CG, Harun N, Powell MP \& Baxley EG (2006) Rural-urban differences in depression prevalence: implications for family medicine. Health Serv Res 38, 653-660.

2. DeNavas-Walt C, Proctor BD \& Smith J, US Census Bureau (2007) Income, Poverty, and Health Insurance Coverage in the United States: 2006. Washington, DC: US Government Printing Office.

3. Economic Research Service (2005) Rural America at a Glance: 2005. Economic Information Bulletin no. 4. Washington, DC: US Department of Agriculture.

4. US Census (2001) Survey of Income and Program Participation Users' Guide (Supplement to the Technical Documentation), 3rd ed. Washington, DC: US Department of Commerce, Economic and Statistics Administration.

5. Economic Research Service (2003) Food security in the United States: measuring food security. http://www.ers. usda.gov/Briefing/FoodSecurity/measurement (accessed November 2003).

6. Nord M, Andrews M \& Carlson S (2007) Household Food Security in the United States, 2006. Economic Research Report no. 49. Washington, DC: US Department of Agriculture.

7. US Department of Agriculture (1999) The rural poor's access to supermarkets and large grocery stores. Fam Econ Nutr Rev 12, 90-99.

8. Wolkwitz K (2007) Technical Appendices to Trends in Food Stamp Participation Rates: 1999 to 2005: Final Report. Report no. 6044-409. Princeton, NJ: Mathematica Policy Research, Inc.

9. Kaiser L, Baumrind N \& Dumbauld S (2007) Who is foodinsecure in California? Findings from the California Women's Health Survey, 2004. Public Health Nutr 10, 574-581.

10. Chilton M \& Booth S (2007) Hunger of the body and hunger of the mind: African American women's perceptions of food insecurity, health and violence. J Nutr Educ Behav 39, 116-125.

11. Casey P, Goolsby S, Berkowitz C et al.; Children's Sentinel Nutritional Assessment Program Study Group (2004) Maternal depression, changing public assistance, food security, and child health status. Pediatrics 113, 298-304.
12. Heflin CM, Siefert K \& Williams DR (2005) Food insufficiency and women's mental health: findings from a 3-year panel of welfare recipients. Soc Sci Med 61, 1971-1982.

13. Siefert K, Heflin CM, Corcoran ME \& Williams DR (2001) Food insufficiency and the physical and mental health of low-income women. Women Health 32, 159-177.

14. Siefert K, Heflin CM, Corcoran ME \& Williams DR (2004) Food insufficiency and physical and mental health in a longitudinal survey of welfare recipients. $J$ Health Soc Behav 45, 171-186.

15. Hauenstein EJ, Petterson S, Merwin E, Rovnyak V, Heise B \& Wagner D (2006) Rurality, gender, and mental health treatment. Fam Community Health 29, 169-185.

16. Hauenstein EJ \& Boyd MR (1994) Depressive symptoms in young women of Piedmont: prevalence in rural women. Women Health 21, 105-123.

17. Sears SF, Danda CE \& Evans GD (1999) PRIME-MD and rural primary care: detecting depression in a low-income rural population. Prof Psychol Res Pr 30, 357-360.

18. Wijinberg M \& Reding K (1999) Reclaiming a stress focus: the hassels of rural, poor single mothers. Fam Soc 80, 506-515.

19. Brown AC, Brody GH \& Stoneman Z (2000) Rural black women and depression: a contextual analysis. J Marriage Fam 62, 187-198.

20. Carruth AK \& Logan CA (2002) Depressive symptoms in farm women: effects of health status and farming lifestyle characteristics, behaviors, and beliefs. J Community Health 27, 213-228.

21. Hauenstein EJ \& Peddada SD (2007) Prevalence of major depressive episodes in rural women using primary care. J Health Care Poor Underserved 18, 185-202.

22. Simmons LA, Huddleston-Casas CA \& Berry A (2007) Low-income rural women and depression: factors associated with self-reporting. Am J Health Behav 31, 657-666.

23. Butler MA \& Beale C (1994) Rural-Urban Continuum Codes for Metro and Nonmetro Counties, 1993. Report no. 9425. Washington, DC: US Government Printing Office.

24. Hamilton WL, Cook JT \& Thompson WW (1997) Household Food Insecurity in the United States in 1995: Summary Report of the Food Security Measurement Project. Alexandria, VA: Food and Consumer Service, US Department of Agriculture.

25. Frongillo EA (1999) Validation of measures of food insecurity and hunger. J Nutr 129, 506S-509S.

26. Radloff LS (1977) The CES-D scale: a self-report depression scale for research in the general population. Appl Psychol Meas 1, 385-401.

27. Simmons LA, Braun B, Charnigo R, Havens JR \& Wright DW (2008) Depression and poverty among rural women: a relationship of social causation or social selection? J Rural Health 24, 292-298.

28. Bollen KA \& Long JS (editors) (1993) Testing Structural Equation Models. Newbury Park, CA: Sage Publications.

29. Mueller RO (1996) Basic Principles of Structural Equation Modeling. New York, NY: Springer-Verlag.

30. Hvas AM, Juul S, Bech P \& Nexo E (2004) Vitamin B 6 level is associated with symptoms of depression. Psychother Psychosom 73, 340-343.

31. Alpert JE \& Fava M (1997) Nutrition and depression: the role of folate. Nutr Rev 55, 145-149.

32. Alpert JE, Mischoulon D, Nierenberg AA \& Fava M (2000) Nutrition and depression: focus on folate. Nutrition 16, $544-581$

33. Spoth R (1997) Challenges in defining and developing the field of rural mental disorder preventive intervention research. Am J Community Psychol 25, 425-448. 
34. Olson AL, Dietrich AJ, Prazar G \& Hurley J (2006) Brief maternal depression screening at well-child visits. Pediatrics 118, 207-216.

35. Arons B (2002) Mental health services in rural America. Vital Speeches Day 66, 369-371.

36. Rost K, Smith R \& Taylor J (1993) Rural-urban differences in stigma and the use of care for depressive disorders. J Rural Health 9, 57-62.
37. Hauenstein EJ (2003) No comfort in the rural south: women living depressed. Arch Psychiatr Nurs 17, 3-11.

38. Hoyt D, Conger R, Valde J \& Weihs K (1997) Psychological distress and help seeking in rural America. Am J Community Psychol 25, 449-470.

39. Kelleher KJ \& Robbins JM (1997) Social and economic consequences of rural alcohol use. NIDA Res Monogr $\mathbf{1 6 8}$, 196-219. 